\title{
De la Pedagogía conceptual y los niveles de lectura de un grupo de la LEIyP, UPN- México
}

\author{
DOI: $10.46932 / \mathrm{sfjdv2n1-040}$
}

Received in: November 1st, 2020

Accepted in: December 30th, 2020

\author{
Karina Rodríguez Cortés \\ Universidad Pedagógica Nacional, Unidad 097 (México) \\ E-mail: krodriguez@g.upn.mx \\ Juana Josefa Ruíz Cruz \\ Universidad Pedagógica Nacional, Unidad 095 (México) \\ E-mail: juanisruiz@ hotmail.com \\ Celia Reyes Anaya \\ Universidad Pedagógica Nacional, Unidad 281 (México) \\ E-mail: creyesa21@gmail.com
}

\section{RESUMEN}

La ponencia tiene como propósito, analizar el itinerario que han definido 21 estudiantes de la Licenciatura en educación Inicial y preescolar (LEIyP), que oferta a nivel nacional la Universidad Pedagógica Nacional en México, para alcanzar los niveles de lectura categorial y metasemántico. El trabajo contiene tres apartados; el primero, presenta de manera general el contexto en que se realiza la investigación; el segundo, contiene el sustento teórico de la pedagogía conceptual para la enseñanza de la lectura y escritura que integra el Módulo de Lectura Temprana (parte del catálogo de los módulos de la LEIyP, en modalidad mixta); y el tercero, sistematiza los resultados de los trabajos que las estudiantes presentan, a partir de los niveles de lectura que define Miguel de Zubiría Samper. Y a manera de cierre, se integran las conclusiones.

Palabras clave: pedagogía conceptual, niveles de lectura, lectura categorial, lectura semántica.

\section{INTRODUCCIÓN}

La enseñanza y el aprendizaje de la lectura ha ocupado el tiempo de diversos autores desde hace varios siglos. Por ejemplo, para Juan Amos Comenio, el aprendizaje de la lectura tendría que realizarse en la Escuela Común, en ésta, el estudiantado tendría que leer con facilidad y expedición el idioma propio,ya impreso, ya manuscrito; y posteriormente en la Escuela Latina debería ser capaz de expresar los conceptos de todas las cosas, con perfección en idioma patrio y en latín, y lo suficiente en griego y en hebreo. El autor planteaba, para lo que en el siglo XXI corresponde a la educación superior, el aprendizaje de cuatro idiomas.

En la investigación se parte, de que el aprendizaje de la lectura, estávinculado con la oralidad. La adquisición del lenguaje oral se concibe como unproceso cognitivo, emocional, social y cultural (Comenio, Piaget, Condemarín, De Zubiría, Cassany; entre otros), que posibilita primero la lectura, y luego la escritura. Es "la llave de acceso al conocimiento en 
la sociedad de la información" lo que provoca una revolución entendida como la renovación delas prácticas de lectura. Al respecto, los procesos de mediación de la lectura enlas escuelas públicas en México, no han sido afortunados.

En el país se prohibió la reprobación del primer grado de primaria alsegundo para evitar el alto índice de reprobación por la falta del dominio de lalectura fonética, y paradójicamente solamente, en los preescolares particulares, se enseñan la lectura y la escritura en dos idiomas. En la presenteponencia presentamos los resultados del cómo un grupo de profesoras que trabaja con población de 0 a 6 años (y que estudian la Licenciatura de Educación Inicial y Preescolar en la UPN), pueden pasar de un nivel delectura fonética a otro más alto.

\subsection{PROBLEMATIZACIÓN}

Las educadoras que laboran con población infantil de 3 a 6 años en pocas ocasiones consideran los dispositivos de comunicación con los que las nuevas generaciones se adentran pronto a un mundo de imágenes, símbolos, logos, etc.; y que podrían ser de utilidad para enseñar a edades tempranas la lectura, siempre y cuando las educadoras y agentes educativos cuenten con un referente teórico sobre las operaciones mentales que están presentes en el aprendizaje de lectura desde los primeros años de vida y hasta la Universidad.

Con base en lo anterior, nos hemos planteado las siguientes preguntas:

1. ¿Cuál es el nivel de lectura en que se encuentran las educadorasque cursan el Módulo "Lectura Temprana"?

2. ¿Contiene el itinerario que las educadoras construyeron unaprendizaje gradual de la lectura como lo plantea Miguel de Zubiría Samper en la Teoría de los seis lecturas?

\section{DE LA PEDAGOGÍA CONCEPTUAL A LA TEORÍA DE LOS SEIS NIVELES DELECTURA}

La Pedagogía Conceptual es una teoría desarrollada en Colombia desde los años ochenta del siglo XX, por un equipo de investigadores de la Fundación Alberto Merani para el Desarrollo de la Inteligencia. El propósito educativo del planteamiento está orientado a la activación de las operaciones de pensamiento, para dar paso al desarrollo de los procesos intelectuales valorativos de los individuos en general. En tal sentido, la lectura está centrada en el análisis de formas superiores del "acto-lector", indicando las operaciones que participan en la comprensión de textos escritos. 
La pedagogía conceptual parte de un desarrollo, que comienza por precogniciones construidas en un proceso, que privilegia la experiencia cognoscitiva en términos cualitativos. Desde la perspectiva de Miguel de Zubiría Samper "La pedagogía actual no ha percibido el complejo conjunto deoperaciones que se suceden en la mente del alumno, ni la variedad de conceptos y relaciones que implican el acceso a conocimientos más sencillos, porque al privilegiar el conocimiento exacto ha obligado a los estudiantes a recurrir a la memorización, que mal que bien, le han permitido desenvolverse en el ámbito de los aprendizajes en el corto plazo" (De Zubiría, 1996, pág.24).

Desde la Pedagogía Conceptual se distinguen seis niveles de lectura, que van desde las más elementales hasta las más complejas (desde el preescolar hasta la universidad). El primer nivel de la lectura, fonético, permite establecer relación entre el grafema y el fonema. Miguel de Zubiría Samper explica que emplea las operaciones de análisis y síntesis: “Obliga al cerebro reconocer patrones complejos de palabras, a reconocer la palabras como unidades" (De Zubiría, 1996: 16); la polémica sobre los “métodos” (global y sintético) ha pasado a ocupar un segundo lugar. Sin que esto signifique que a la fecha exista consenso respecto a una sola forma de enseñar a leer y escribir.

El segundo nivel de lectura es la decodificación primaria, cuyo objeto es la "comprensión" lectora, y consiste en traducir, interpretar y convertir las palabras en conceptos. Permite establecer relaciones entre la palabra y formar nociones o frases. Utiliza mecanismos como la recuperación léxica, la sinonimía, la antonimía y la radicación. El tercer nivel o decodificación secundaria, comprende un conjunto de operaciones mentales cuya función es extraer proposiciones e interpretarlos por medio de análisis. Permiteestablecer relación entre oración y las proposiciones y utiliza mecanismos como la puntuación, la pronominalización y la inferencia. El cuarto nivel o decodificación terciaria, se centra en encontrar las macroproposiciones, descubrir las relaciones lógicas, temporales, espaciales en referencia a la idea mayor o tesis. Permite establecer relaciones entre el texto y su respectiva estructura semántica.

El quinto nivel o lectura categorial es la manera de descomponer un textoen sus tesis, proposiciones e identificar la estructura categorial, utiliza todas las herramientas y los instrumentos del pensamiento. Y el último nivel, según Miguel De Zubiría es la lectura metasemántica, ésta permite comparar, establecer analogías y hacer correspondencias con otros sistemas. Su finalidades contrastar, ir más allá de las circunstancias socioculturales en que está expresado el texto y someterlo a la crítica (Ver Esquema 1). 
Esquema 1. La Lectura desde la Pedagogía Conceptual.
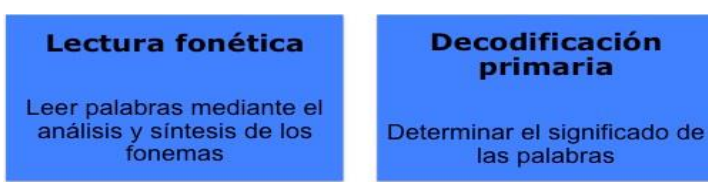

Decodificación terciaria

Encontrar la lectura ásica de ideas del tex

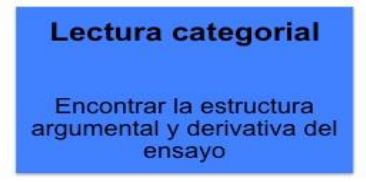

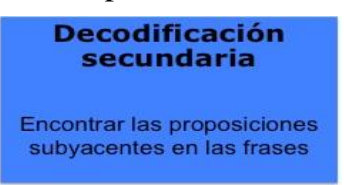

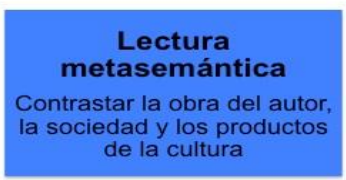

En síntesis "el procesamiento fonético, elemental, es de naturaleza perceptual analítico-sintética, habrían de ocurrir, sí se enseñan a los niños y jóvenes, cinco tipos adicionales ascendentes de procesamiento, denominados en conjunto: decodificación. Primaria (palabras), secundaria (frases), terciaria (párrafos), categorial y por último, metasemántica (análisis transtextual)...”(De Zubiría, 1996, pág. 56).

De acuerdo con Mabel Condemarín (1989), integrar tempranamente lalectura en el nivel del Jardín de niños, es un tema que ha suscitado más puntos de vista en contra que a favor. Los argumentos que se esgrimen, se apoyan porlo general, más en la intuición que en la investigación y la práctica, y muchas afirmaciones tienden a constituirse "en mitos que se trasmiten de una a otra generación de educadores y de padres". Para el caso de México, en lasprácticas educativas en los preescolares particulares, las y los niñas (os)aprenden a leer y escribir antes de ingresar a primer grado, abriendo una brecha educativa considerable entre las instituciones públicas y privadas. Lo anterior, sin considerar que en algunas instituciones incluso los infantes aprenden simultáneamente a la lectura y la escritura de dos idiomas.

En el Módulo Lectura temprana, que forma parte de la malla curricular dela LEIyP, se analizan y llevan a la práctica las propuestas de Mabel Condemarín y Miguel de Zubiría Samper para que la lectura sea un proceso intencionado y mediado desde el preescolar. Tiene como objetivo: integrar en el trabajo educativo de las educadoras una mediación para el aprendizaje de lalectura desde los 4 años; y está estructurado en cuatro bloques: el primero, Aprendizaje de la lectura desde la pedagogía conceptual; el segundo, Lectura fonética: análisis y síntesis; el tercero, ambientes letrados; y el cuarto, Proyecto educativo para mediar el aprendizaje la lectura en preescolar.

En la ponencia solamente se presenta lo correspondiente al Bloque I. Aprendizaje de la lectura desde la Pedagogía Conceptual, que tiene como propósito formativo:

- Analizar el planteamiento relacionado con el aprendizaje de la lectura desde la denominada Pedagogía Conceptual para evaluar en el nivel de dominio en que se encuentran las participantes en el Módulo. 
Los contenidos temáticos son:

1. Concepciones sobre el aprendizaje de la lectura en preescolar

2. Pedagogía conceptual y aprendizaje de la lectura

3. Autoevaluación de los niveles de lectura y definición de itinerario pa-ra seguir aprendiendo a leer.

La secuencia didáctica instrumentada en Moodle es la siguiente:

\begin{tabular}{|c|c|}
\hline Secuencia didáctica & Producto esperado \\
\hline $\begin{array}{l}\text { 1. Leer el artículo de Mabel Condemarín: } \\
\text { "Iniciación temprana en la lectura: interrogantes y } \\
\text { retos" y elaborar dos láminas de power point: una } \\
\text { que sintetice la postura de la autora; y otra, con las } \\
\text { estrategias que propone para el aprendizaje de la } \\
\text { lectura en preescolar. }\end{array}$ & Dos láminas de powerpoint. \\
\hline $\begin{array}{l}\text { Subir a un foro las láminas y retroalimentar el } \\
\text { trabajo dos colegas. }\end{array}$ & $\begin{array}{l}\text { Escrito de } 1 \text { cuartilla que } \\
\text { defina a la "Pedagogía } \\
\text { Conceptual" }\end{array}$ \\
\hline 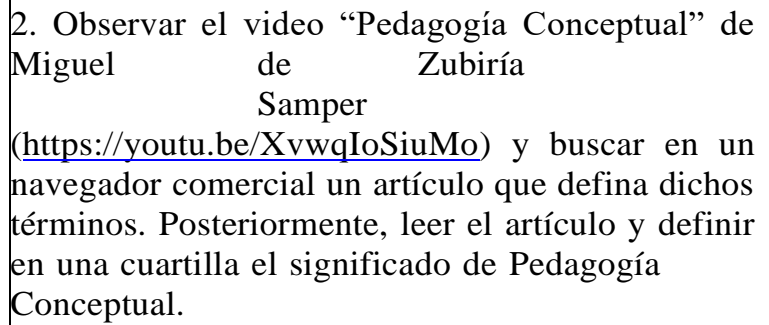 & \\
\hline $\begin{array}{l}\text { 3. Leer "Los seis niveles de lectura" las pp. 55- } \\
74 \text { del libro Teoría de las seis lecturas, tomo. I, y } \\
\text { observar el vídeo diseño de un cuestionario } \\
\text { (https://youtu.be/HT8oxgUDSOU); y con base en } \\
\text { éstos, diseñar un cuestionario para evaluar los } \\
\text { niveles de lectura. }\end{array}$ & $\begin{array}{l}\text { Cuestionario } \\
\text { aautoevaluar los niveles de } \\
\text { lectura }\end{array}$ \\
\hline $\begin{array}{l}\text { Aplicar el cuestionario para autoevaluar el nivelde } \\
\text { lectura del participante. } \\
\text { 4. Presentar los resultados de su autoevaluación en } \\
\text { torno al nivel de lectura en que se y elaborar un } \\
\text { itinerario sobre cómo podría seguir aprendiendo a } \\
\text { leer, con base en la propuesta de Miguel de Zubiría } \\
\text { Samper. }\end{array}$ & $\begin{array}{l}\text { Itinerario para seguir } \\
\text { aprendiendo a leer }\end{array}$ \\
\hline
\end{tabular}

\section{LOS RESULTADOS}

Primero interesa destacar, que las 21 participantes en el Módulo Lectura Temprana, de octubre de 2017 a febrero de 2018, construyeron un instrumento con base en la teoría de las seis lectura (categorías) y autoevaluaron su nivel de Lectura. Segundo, que con el propósito de mantener el anonimato a cada estudiante se le asignó un número y se 
identificaron 8 casos sin itinerario de lectura. Y tercero, a continuación se presentan los resultados de manera textual.

\subsection{CATEGORÍA: LECTURA FONÉTICA}

Las dos operaciones mentales que se desarrollan en la Lectura fonética son el análisis y la síntesis, a partir de la composición y descomposicón de palabras, con sentido o sin sentido. Y ampliar el léxico. En el itinerario construido por las participantes solamente se apegan a ésta son las siguientes:

- A9 Repetición de palabras que nos cueste pronunciar para después poder leerla más rápidamente. Continuar repitiendo y poco a poco pronunciar palabras más complejas y largas. Fomentarnos a nosotras mismas con la lectura comprar libros.

- A9. El mejor método para aumentar la fluidez de los principiantes consistirá en repetir determinada lectura. Cuando se repite cierta lec- tura, se mejora la precisión, esto ayuda con las palabras complicadas y poco a poco se expresara una lectura fluida.

- A10 Lecturas diversas del mundo social y natural. Me apropiaré de estrategias de lectura como: relacionar, registrar, formular etc.

- A14 Conocer completamente el alfabeto. Corregir la fonética cuando así se requiera. Respetar los signos de puntuación.

- A17 Buscar pistas en las imágenes. Observar las fotografías o ilustra- ciones de la página. Explorar lo que veo en ellas (personas, lugares, objetos, acciones) para ayudarme a darle sentido a la oración. Decir los sonidos de las palabras. Comenzar por la primera, decir en voz al- ta y lentamente el sonido de cada letra. Luego, repetir los sonidos e intentar mezclarlos para formar la palabra y observar si esa palabra tiene sentido dentro de la oración. Desarmar las palabras. Observar la palabra e intentar reconocer algún sonido, símbolo, prefijo, sufijo, fi- nal o base de palabras que ya conozca. Leer cada "parte" de la palabra por sí sola y luego intentar combinar esas partes y pronunciar la pala- bra en voz alta.

- A17 Seguir leyendo. En lugar de estancarme con alguna palabra que no conozco, seguir leyendo y busca otras pistas. Si la palabra aparece nuevamente en el texto, comparar esa oración con la anterior e inten- tar pensar qué palabra sería la apropiada para darle sentido a ambas.

- A18. Durante al menos 10 minutos en voz alta leer un texto que con- sidere 
significativo. Puede proponer textos breves o continuar un tex- to largo a través de sus clases.

- A18 Leer para conocer y estudiar Transmitir modos de leer propios decada disciplina y manejar los materiales de estudio de cada campo de saber. Practicar en espacios ampliados de aprendizaje leyendo en voz alta, para establecer relaciones entre diferentes materiales. Esta lectu- ra compartida permite detenerse en las dificultades (diferenciar in- formación relevante y secundaria; distinguir causa y consecuencia, aserción y posibilidad, opinión y fundamentación, hechos y opiniones y comprender el léxico de cada disciplina). Implementar prácticas breves de lectura individual silenciosa y luego compartir interpreta- ciones.

\subsection{CATEGORÍA: DECODIFICACIÓN PRIMARIA}

En este nivel de lectura se desarrollan cuatro suboperaciones: la sinonimía, la antonimía y la radicación. Los itinerarios que se apegan a ésta son:

- A1 "cuando estemos leyendo y encontremos palabras que no sepamossu significado, busquémoslo y elaborar un glosario con ellas anotando lo que nosotros hemos comprendido de cada una".

- A9. Con temas de nuestro interés, sacar ideas principales por hojas o por párrafos para ir comprendiendo poco apoco el mensaje de cada uno de estos párrafos. Ejercicios para agilizar nuestra mente es decir ejercicios de memoria. Leer lo que más se pueda y que varíe el tipo de texto. Cuando se lea, subrayar aquellas palabras que se desconoz- can, luego, buscarlas en el diccionario.

- A10 Pondré mi lupa en las palabras. aprendere a leer y escribir con progresiva autonomía: buscaré significados de palabras y la reflexión de las mismas.

- A10 Circulos de Estudio. leer para otros, conocer poesias, fabulas, obras teatrales y novelas. compartiré la lectura con otros, pondré en juego la memoria de la historia y recuperaré información.

- A12 Siempre que vea una palabra nueva o que no entienda buscar el significado.

- A13 Practicar diariamente ejercicios con cada suboperador con dife- rentes tipos de texto.

- A14. Buscar el significado de palabras que no sean familiares.

- A17 Buscar palabras que NO comprenda. Deducir el significado y la pronunciación 
de las palabras Buscar conexiones Observar si alguna palabra desconocida se asemeja a otra que me resulte familiar.

- A17 Releer. Leer nuevamente la oración. Intentar reemplazar distintaspalabras por la palabra que no conozco y verificar si la oración tiene sentido.

\subsection{CATEGORÍA: DECODIFICACIÓN SECUNDARIA}

Establece la relación entre oración y las proposiciones y utiliza mecanismos como la puntuación, la pronominalización y la inferencia. Ninguna de las respuestas presenta de manera explícita cómo trabajaría cada una de las suboperaciones que subyacen en la categoría.

\subsection{CATEGORÍA: DECODIFICACIÓN TERCIARIA}

Centrada en encontrar las macroproposiciones, descubrir las relaciones lógicas, temporales, espaciales en referencia a la idea mayor o tesis.

- A13 Identificar macroproposiciones estructuras semánticas y modelosen diversos textos al menos dos veces por semana. No explícita el cómo, solamente repite de manera textual lo propuesto por el autor.

- A14 Círculos de lectura. Releer los textos para entender el sentido de la lectura.

- A16 Ir realizando notas del esquema de sistema de proposiciones. Tampoco explícita el cómo.

En las categorías de lectura categorial y lectura metasemántica, ninguna de los itinerarios presenta de manera explícita cómo trabajaría la contrastaciónde teorías con otras. Por último, en la tabla 1 se presenta la síntesis de los niveles presentados en los itinerario para seguir desarrollando los niveles de lectura:

Tabla 1. Niveles de lectura de un grupo de la LEIyP

\begin{tabular}{|c|c|c|c|l|l|}
\hline $\begin{array}{l}\text { Lectura } \\
\text { Fonética }\end{array}$ & $\begin{array}{c}\text { Decodificación } \\
\text { Primaria }\end{array}$ & DS* & $\begin{array}{c}\text { Decodificación } \\
\text { Terciaria }\end{array}$ & LC** & L $* * *$ \\
\hline A 9 (2) & A1 & A13 & & \\
\hline A10 & A9 & A 14 & & \\
\hline A14 & A10 (2) & & A16 & & \\
\hline A $17(2)$ & A12 & & & & \\
\hline A18 (2) & A 13 & & & & \\
\hline & A 14 & & & & \\
\hline & A 17 & & & & \\
\hline
\end{tabular}


Decodificación Secundaria

** Lectura Categorial

**** Lectura Metasemántica

Como se anota al inicio del análisis, 8 de 21 participantes no comprendieron la indicación. Y de la tabla y la reproducción textual de los itinerarios lo que se observa es que 5 estudiantes del Módulo "Lectura temprana" saben cómo podrían seguir desarrollando la lectura fonética y 7 la decodificación primaria, a partir de ésta, se presenta un salto hasta la decodificación terciaria. Y de las dos últimas categorías, sí bien la incluyeron en sus itinerarios, generalmente presentaban textualmente la definición del autor, pero no el cómo podrían llevarla a la practica para desarrollarlas.

\section{CONCLUSIONES}

La tabla y la reproducción textual de los itinerarios reflejan el nivel de lectura en que las estudiantes del Módulo "Lectura temprana" se encuentran:

- 8 casos alcanzan la categoría de Lectura fonética, dado que no comprendieron la indicación para integrar el producto final del bloque 1: un itinerario para seguir aprendiendo a leer.

- 13 en tres categorías decodificación primaria, secundaria y terciaria.

- A10 aparece en la categoría de decodificación primaria y secundaria.

- 1 en el nivel de Lectura categorial, ya que la escritura contempla otros procesos más complejos,

- A1, A16 y A18 coinciden en el itinerario más completo de escritura.

La reproducción textual de los "trozos de los itinerario", también nospermitió ubicar algunas faltas de ortografía y copias textuales de las definiciones de los niveles de lectura que dan cuenta de lo que Miguel de Zubiría Samper y Mabel Condemarín sostienen: el proceso lector tiene que seracompañado desde el preescolar hasta la educación superior. Y a partir de las dos preguntas planteadas en la problematización y del análisis realizado podemos derivar las siguientes conclusiones:

- La modalidad en línea presenta el mismo problema que la educa- ción presencial, la no comprensión de lo que se indica como tareaa ser elaborada, a pesar de que en la 
primera se presenta por escri-to.

- La transferencia de lo conceptual a la práctica educativa, sola- mente lo alcanzaron 9 de las participantes, en dos de los primeros niveles y una en el nivel de decodificación terciaria.

- El primer nivel de lectura es el que tiene que ser desarrollado enel nivel preescolar, en este sentido, el propósito de la actividad lo alcanzó casi el 50\% de las participantes (A1, A9, A 10, A 12, A13, A14, A 16, A 17 y A 18).

- Los itinerarios de 4 participantes integraron las definiciones delos niveles de lectura, pero no lograron transferir lo conceptual a estrategias o actividades para el desarrollo de éstos.

Los niveles más elevados de lectura, requerían que las estudiantes practicaran los tres primeros niveles de lectura para comprender que los dos últimos niveles corresponden a la lectura de teorías y contrastación deéstas con otras. 


\section{REFERENCIAS}

Condemarín, M. (1989). "Iniciación temprana en la lectura: interrogantes y retos". Condemarín, M. (1989). Lectura temprana (Jardin de niños y primer grado).

Santiago, Chile: Andrés Bello.

De Zubiría, M. (1996). Pedagogía Conceptual. Bogotá, Colombia: Fundación Alberto Merani para el Desarrollo de la Inteligencia.

De Zubiría, M. (1997). Teoría de la seis lecturas I. (F. A. Inteligencia, Ed.)Bogotá, Colombia. Pang, E. (2003). La enseñanza de la Lectura. (CENEVAL, Ed.) CDMX, México.

Bibliografía

Argudín, Y. (1995). Aprender a pensar leyendo bien. Habilidades de lectura anivel superior. México: Plaza y Valdez.

Condemarín, M. (1989). "Iniciación temprana en la lectura: interrogantes y retos". Condemarín, M. (1989). Lectura temprana (Jardin de niños y primer grado).

Santiago, Chile: Andrés Bello.

Comenius, J. A. (1986). Didáctica Magana. Madrid, España: Akal.

Creme, P., \& Lea, M. Escribir en la Universidad. Barcelona, España: Gedisa.

De Zubiría, M. (1996). Pedagogía Conceptual. Bogotá, Colombia: Fundación Alberto Merani para el Desarrollo de la Inteligencia.

De Zubiría, M. (1997). Teoría de la seis lecturas I. (F. A. Inteligencia, Ed.)Bogotá, Colombia.

De Zubiría, M. (1998). Teoría de las seis lecturas II. Bogotá, Colombia: Fundación Alberto Merani para el Desarrollo de la Inteligencia.

MCEP. (S/F). El aprendizaje de la lectura mediante el método global. España:Movimiento Cooperativo de Escuela Popular/Grupo territorial Huelva.

Pang, E. (2003). La enseñanza de la Lectura. (CENEVAL, Ed.) CDMX, México. Piaget, J. (1991). Seis Estudios de Psicología. Barcelona, España: Labor. 